\title{
Réseaux canadiens de recherche sur les vaccins : ressources sur la sécurité des vaccins pour le Canada
}

\section{McCarthy $\mathrm{J}^{1}$, Halperin $\mathrm{SA}^{1,2^{\star}}$, Bettinger $\mathrm{JA}^{3,4}$, Langley $\mathrm{JM}^{1,2}$, Crowcroft NS ${ }^{5,6}$, Deeks $\mathrm{S}^{5,6}$, Kwong $\mathrm{JC}^{5-7}$, De Serres $\mathrm{G}^{8,9}$, Top $\mathrm{K}^{1,2}$, McNeil $\mathrm{S}^{1,10}$, Scheifele $\mathrm{DW}^{3}$, au nom des investigateurs du Réseau canadien de recherche sur l'immunisation (RCRI)}

${ }^{1}$ Centre canadien de vaccinologie, Université Dalhousie, Centre de soins de santé IWK et Capital Health, Halifax (NouvelleÉcosse).

${ }^{2}$ Département de pédiatrie, Université Dalhousie, Halifax (Nouvelle-Écosse)

${ }^{3}$ Département de pédiatrie, Institut de recherche sur l'enfant et la famille, Université de la Colombie-Britannique, Vancouver (Colombie-Britannique)

${ }^{4}$ Centre d'évaluation des vaccins, BC Children's Hospital et Université de la Colombie-Britannique, Vancouver (ColombieBritannique)

${ }^{5}$ Santé publique-Ontario, Toronto (Ontario)

${ }^{6}$ Dalla Lana School of Public Health, Université de Toronto, Toronto (Ontario)

${ }^{7}$ Institut des sciences d'évaluation clinique, Toronto (Ontario)

${ }^{8}$ Faculté de médecine, Université Laval, Québec (Québec)

${ }^{9}$ Institut national de santé publique du Québec, Montréal (Québec)

${ }^{10}$ Faculté de médecine, Université Dalhousie, Halifax (Nouvelle-Écosse)

*Auteur-ressource : scott.halperin@dal.ca

\section{Résumé}

Le Réseau de recherche sur l'influenza de l'Agence de la santé publique du Canada et des Instituts de recherche en santé du Canada (PCIRN), créé en 2009 dans le but de mener des recherches évaluatives afin d'éclairer la prise de décision dans le domaine de la santé publique, cède la place au Réseau canadien de recherche sur l'immunisation (RCRI), qui reprend le même mandat en l'élargissant à tous les vaccins, dont le vaccin antigrippal. Le RCRI regroupe plusieurs réseaux ayant pour vocation principale de mener des recherches dans le domaine de la sécurité des vaccins, des effets secondaires suivant l'immunisation (ESSI), de la réticence face à la vaccination, de l'efficacité des vaccins et de la couverture vaccinale. Ces réseaux comprennent : un réseau de réalisation d'essais cliniques, un réseau de laboratoires, un réseau de recherche en modélisation et en économie, un réseau de chercheurs spécialisés dans les sciences sociales et humaines, un réseau de surveillance de la sécurité des vaccins, un réseau de surveillance en milieu hospitalier, un réseau de cliniques chargées d'évaluer les ESSI graves et un réseau reliant les capacités de recherche vaccinale des organismes et services de santé publique en place dans les provinces. Le PCIRN a apporté son soutien au système de surveillance de la sécurité des vaccins du Canada et a facilité l'application des résultats de la recherche sur la sécurité vaccinale à l'élaboration de politiques. La surveillance de la sécurité des vaccins et la recherche dans le domaine de la sécurité vaccinale demeureront une priorité pour le tout nouveau Réseau canadien de recherche sur l'immunisation.

\section{Introduction}

Le Réseau de recherche sur l'influenza de l'Agence de la santé publique du Canada (ASPC) et des Instituts de recherche en santé du Canada (IRSC) (PCIRN) avait pour mission de consolider les acquis en matière d'évaluation des vaccins antigrippaux, d'accroître la capacité à tester rapidement les vaccins antigrippaux candidats, de développer des liens entre les chercheurs et les décideurs et de former des chercheurs spécialistes de la grippe. En 2013, l'ASPC et l'IRSC ont annoncé leur décision de financer la création d'un Réseau canadien de recherche sur l'immunisation (RCRI) ayant pour mandat de mener des recherches évaluatives sur les vaccins, notamment sur le vaccin antigrippal objet des efforts de recherche du PCIRN. Actuellement, le RCRI représente plus de 100 investigateurs venant de plus de 40 établissements canadiens, regroupant des experts spécialisés dans la recherche évaluative sur les vaccins, issus de disciplines variées. L'objectif du présent article est de 
décrire la contribution apportée par le PCRIN à l'acquisition de connaissances en matière de sécurité des vaccins dans le passé et de voir comment le RCRI prendra le relais pour élargir ce mandat à l'avenir.

\section{Aperçu du PCIRN et présentation des réseaux de recherche}

Le PCIRN a été conçu en tant que « réseau de réseaux ». Depuis sa création en 2009, ce réseau a traité d'importantes questions ayant trait aux programmes de vaccination contre la grippe.

\section{Réseau de réalisation d'essais cliniques}

Le Réseau de réalisation d'essais cliniques, dont les centres sont répartis à Vancouver, Calgary, Winnipeg, Sudbury, Hamilton, Toronto, Ottawa, Montréal, Québec et Halifax, a la capacité de conduire, sur des groupes de grande taille et des groupes spécialisés, des essais cliniques rapides axés sur la sécurité des vaccins, l'immunogénicité et les mécanismes de l'immunité.

\section{Réseau national canadien d'évaluation de la sécurité des vaccins (CANVAS)}

Le Réseau national canadien d'évaluation de la sécurité des vaccins (CANVAS) collecte et analyse rapidement les données de sécurité recueillies sur des milliers de personnes vaccinées (adultes et enfants) afin de fournir des renseignements sur la sécurité des vaccins antigrippaux aux autorités sanitaires avant les semaines de base de la campagne annuelle de vaccination contre la grippe. Les participants sont recrutés auprès de cliniques de soins actifs et de cliniques de vaccination antigrippale de la santé publique dans cinq provinces. Ils reçoivent un bref sondage électronique huit jours après l'administration du vaccin. Le principal critère d'évaluation est la survenue de tout nouveau problème de santé ou l'aggravation d'une maladie existante qui est suffisamment grave pour engendrer l'absentéisme au travail ou à l'école, entraver les activités quotidiennes ou nécessiter une consultation médicale. En cas de signalement d'événements graves, un suivi par téléphone est effectué pour obtenir de plus amples détails. Un groupe témoin de participants non vaccinés est également recruté chaque année pour déterminer la fréquence de signalement d'événements dans un groupe non vacciné (la fréquence de référence).

\section{Réseau de cliniques de vaccination spéciales du RCRI}

Le Réseau de cliniques de vaccination spéciales est un réseau national établi dans 13 hôpitaux du Canada regroupant des cliniciens experts qui reçoivent des patients individuels ayant subi des effets secondaires suivant l'immunisation (ESSI) ou présentant d'autres problèmes liés à la sécurité des vaccins que leur envoient des professionnels de la santé publique ou des cliniciens. Ce réseau a pour premier objectif de mettre en place des normes de soins cliniques et des pratiques exemplaires pour la prise en charge de ces patients. La normalisation des soins cliniques favorise la réalisation d'études observationnelles. Son deuxième objectif est d'établir une plate-forme de recherche pour la réalisation d'études sur les questions afférentes à la sécurité des vaccins.

\section{Réseau de surveillance des cas graves (SOS)}

Le Réseau de surveillance des cas graves (SOS) assure une surveillance active, chez des adultes canadiens, des cas de grippe nécessitant une hospitalisation. Cette surveillance couvre plus de 18000 lits répartis dans 45 hôpitaux de 7 provinces. Le réseau SOS a pour mission de mesurer le fardeau de la maladie et d'évaluer l'efficacité des vaccins en ce qui concerne la prévention des hospitalisations et des décès liés à la grippe.

\section{Réseau de mise en ouvre et d'évaluation des programmes de vaccination}

Le Réseau de mise en œuvre et d'évaluation des programmes de vaccination mène des études de recherche appliquée, dans le domaine de la santé publique, afférentes à l'administration des vaccins contre la grippe. Les travaux de ce réseau prendront fin au terme de la période de financement du PCIRN.

\section{Réseau de laboratoires de référence}

Le Réseau de laboratoires de référence, qui regroupe cinq laboratoires sur l'ensemble du pays, est géré par des scientifiques compétents dans les domaines de la microbiologie, de la grippe et d'autres maladies infectieuses. Chaque scientifique dirige des programmes de recherche active au sein de son établissement tout en travaillant également pour le compte du secteur de la santé publique. Le réseau de laboratoires soutient le PCIRN dans ses activités d'analyse liées à la grippe et gère la banque de tissus biologiques du réseau. 
Compte rendu des études sur la sécurité des vaccins réalisées par le PCIRN

Si le mandat de recherche sur la grippe du PCIRN est vaste, la recherche sur la sécurité des vaccins a été une priorité pour plusieurs de ses réseaux. À ce jour, le Réseau de réalisation d'essais cliniques a accompli huit essais cliniques, dont un grand nombre étaient axés sur la sécurité des vaccins. Les essais cliniques du PCIRN menés en 2009 sur le vaccin contre la grippe pandémique contenant un adjuvant visaient à mesurer les effets indésirables observés chez des groupes de population différents, notamment des adultes sains (1), des populations autochtones (2) et des personnes atteintes d'une infection par le virus de l'immunodéficience humaine (VIH) (3). En 2010, on craignait que l'administration du vaccin inactivé trivalent contre la grippe saisonnière contenant la souche de la grippe pandémique n'augmente le taux d'effets indésirables au point d'injection et d'effets systémiques chez les personnes ayant précédemment reçu le vaccin contre la grippe pandémique H1N1 contenant un adjuvant, en raison de la présence des taux très élevés d'anticorps préexistants. Deux essais cliniques ont été menés, l'un auprès d'adultes et l'autre auprès d'enfants, dès la mise à disponibilité du vaccin. Les résultats de ces essais ont été présentés aux décideurs et aux planificateurs de programmes avant le déploiement des campagnes annuelles de vaccination contre la grippe $(4,5)$. Le PCIRN a également consacré une grande partie de sa recherche sur la sécurité à la vaccination contre la grippe des personnes allergiques aux œufs, les données fournies par le réseau ayant entraîné un profond remaniement des recommandations sur la vaccination $(6,7)$. L'issue fructueuse de ces études sur la sécurité a mené au développement du Réseau de cliniques d'immunisation spéciales qui poursuivra ses travaux sous l'égide du RCRI.

Le Réseau national canadien d'évaluation de la sécurité des vaccins a également contribué à l'accroissement des connaissances sur la sécurité des vaccins contre la grippe au cours de ces quelques dernières années $(8,9)$. Durant la saison 2013-2014, ce réseau a recruté un nombre record de répondants, soit plus de 35000 adultes et parents d'enfants. Le taux de réponse a été de $61 \%(n=13127)$ dans le groupe vacciné et de $50 \%(n=6763)$ dans le groupe témoin. Les parents d'enfants âgés de 6 mois à 16 ans représentaient $12 \%(n=2314)$ des participants. La majorité des participants n'ont signalé aucun effet sur leur santé (96\% des personnes vaccinées et $93 \%$ des témoins). Dans le groupe témoin, $5 \%$ des participants ont signalé un événement ayant entraîné l'absentéisme, entravé les activités quotidiennes ou nécessité une consultation médicale, alors que $2,5 \%$ des participants vaccinés ont signalé ce type d'événements. Les événements restants $(2 \%$ dans le groupe témoin et $1,5 \%$ chez les répondants vaccinés) ont été signalés comme étant gênants ou facilement tolérables. Indépendamment du statut de vaccination, les symptômes les plus souvent signalés chez les adultes et les enfants ont été d'origine respiratoire et gastro-intestinale; ils ont été plus fréquents dans le groupe témoin que chez les participants vaccinés. Chez les enfants, des changements dans les habitudes alimentaires ont été signalés plus souvent dans le groupe vacciné. Aucun décès ni aucune hospitalisation n'ont été signalés dans le groupe témoin ni chez les répondants vaccinés. Moins de $1 \%$ des répondants vaccinés $(0,4 \%)$ et $1 \%$ des témoins ont signalé avoir consulté un médecin pour leurs symptômes; le taux de visites au service des urgences étant identique dans les deux groupes parmi ces répondants (0,53/1 000 participants). Malgré une capacité à détecter des événements survenant à un taux inférieur à 1/1 000, nous n'avons observé aucun signe de problèmes de sécurité associé aux vaccins antigrippaux administrés entre 2013 et 2014, ni chez les adultes, ni chez les enfants. En cas de détection d'un tel signe, le Réseau national canadien d'évaluation de la sécurité des vaccins a la capacité de se mettre en rapport avec les autorités sanitaires et d'effectuer un suivi approfondi.

Après l'autorisation de mise sur le marché du vaccin contre la grippe pandémique avec adjuvant au Canada, en 2009, et durant les campagnes d'immunisation de 2010-2011, le Réseau de surveillance des cas graves a mené une surveillance active axée sur la détection de certains effets secondaires d'un intérêt particulier. Les observateurs chargés de la surveillance dans l'ensemble des hôpitaux participants ont passé en revue, chaque jour, la totalité des admissions aux services médicaux, aux unités de soins intensifs et aux services d'hématologie et de neurologie à la recherche de patients admis pour cause de syndrome de Guillain-Barré, de purpura thrombocytopénique idiopathique (PTT) ou d'encéphalite correspondant aux définitions de cas établies par Brighton Collaboration et dont les symptômes étaient apparus dans les six semaines de l'administration du vaccin antigrippal. Aucun cas d'ESSI n'a été détecté dans les hôpitaux participants au cours de la période de surveillance. 


\section{Aperçu du RCRI et présentation des réseaux}

À l'image du PCIRN, le RCRI sera un « réseau de réseaux ». Étant donné que le financement du PCIRN prend fin en 2015, plusieurs de ses infrastructures de réseau seront intégrées dans le RCRI qui comprendra également plusieurs nouveaux réseaux afin d'être en mesure de répondre aux besoins de son mandat élargi.

L'intégration du réseau de cliniques d'immunisation spéciales au RCRI se fera tout naturellement. Le réseau de cliniques d'immunisation spéciales a été créé durant la période de renouvellement du PCIRN, ayant été conçu pour évaluer les ESSI liés à tous les vaccins, et pas seulement au vaccin antigrippal, selon une méthode normalisée. La première année de sa création, les investigateurs ont passé en revue les publications traitant du risque de récurrence des ESSI et rédigé un guide de prise en charge clinique. Le réseau recrute actuellement des patients pour une étude de base visant à évaluer les risques que présentent la revaccination de patients ayant subi un ESSI et l'administration de vaccins à des patients présentant des affections sous-jacentes susceptibles de constituer une contre-indication manifeste.

Pour le Réseau de réalisation d'essais cliniques, le passage du PCIRN au RCRI ne posera pas de problèmes étant donné que tous les centres possèdent le savoir-faire requis pour mener des essais cliniques sans rapport avec le vaccin antigrippal.

Le Réseau de surveillance des cas graves passera du PCIRN au RCRI, apportant à ce dernier ses activités de surveillance en milieu hospitalier et sa capacité à mesurer l'efficacité des vaccins. Si sa vocation principale était de décrire le fardeau de la grippe et l'efficacité du vaccin antigrippal contre les cas graves de la maladie, ce réseau de surveillance a cependant déjà bénéficié de fonds de l'industrie pour mesurer le fardeau de la méningococcie invasive, de la pneumonie acquise dans la collectivité et de la pneumococcie invasive chez les adultes.

Bien que le Réseau national canadien d'évaluation de la sécurité des vaccins ait été créé à l'origine pour évaluer la sécurité des vaccins dans les suites immédiates de la mise en œuvre des campagnes annuelles de vaccination contre la grippe, il sera fait appel à ses services pour mener des études sur la sécurité d'autres vaccins administrés dans ces cohortes ainsi que pour évaluer la réticence face à la vaccination et l'efficacité des vaccins.

Le Réseau de laboratoires de référence passera du PCIRN au RCRI et continuera d'assurer la gestion active d'une réserve archivée d'échantillons sériques et d'autres échantillons biologiques collectés par les réseaux du $\mathrm{RCRI}$, laquelle réserve sera accessible aux investigateurs pour mener de futures études. Ce réseau comprendra également le Réseau d'évaluation de l'immunité des Canadiens et du risque d'épidémie (iCARE). Le Réseau de laboratoires de référence couvre cinq provinces et coordonne l'appariement des capacités d'analyse des laboratoires universitaires et de santé publique participants aux besoins des projets du RCRI.

\section{Réseau collaboratif provincial}

Le Réseau collaboratif provincial est un nouveau réseau créé par le RCRI dans le but de tirer profit des importantes capacités de recherche des organismes de santé publique et autres services de santé des provinces et de fournir un cadre favorable à la réalisation concertée d'études de recherche appliquée évaluatives et programmatiques dans le domaine de la santé publique. Le réseau mettra au point des protocoles communs pour évaluer la sécurité des vaccins, leur efficacité et la couverture vaccinale, reposant notamment sur l'utilisation des données agrégées et des données individuelles couplables contenues dans diverses bases de données en place au sein de chaque province ( $p$. ex. maladies à déclaration obligatoire, analyses de laboratoire, immunisation, utilisation des soins de santé et statistiques de l'état civil). Cette démarche est analogue à celle du Vaccine Safety Datalink des États-Unis et du Réseau canadien pour l'étude observationnelle des effets des médicaments (10). Actuellement, des investigateurs sont en place dans 7 des 13 provinces/territoires. Nous espérons augmenter ce nombre à l'avenir et consolider le protocole mis au point lors de nos études initiales.

\section{Réseau de recherche en sciences sociales et humaines}

Le Réseau de recherche en sciences sociales et humaines nouvellement créé du RCRI établira un lien entre les spécialistes en sciences sociales et les chercheurs en sciences humaines à l'échelle du pays en vue d'examiner les implications éthiques, juridiques et sociales des programmes de vaccination et des activités de recherche 
menées par le $\mathrm{RCRI}$. Le réseau améliorera la capacité du $\mathrm{RCRI}$ à répondre aux problèmes sociétaux soulevés par tous les projets proposés et desservira les activités de recherche axées sur les sciences sociales et humaines générées par le RCRI; une place majeure sera accordée à la réticence face à la vaccination.

\section{Réseau de recherche en modélisation et en économie (ModERN)}

Le Réseau de recherche en modélisation et en économie du RCRI, de création récente, établira des liens entre les modéliseurs/économistes en matière de santé dans au moins cinq provinces dans le but d'effectuer des analyses épidémiologiques, des exercices de modélisation mathématique et une analyse économique destinée à étudier la rentabilité et l'efficacité pour la population des interventions en santé publique. Le réseau ModERN a également la capacité d'examiner la probabilité des éclosions en se fondant sur la couverture vaccinale, de prédire l'ampleur des effets des pandémies émergentes et les stratégies de contrôle optimales pour le Canada ainsi que d'examiner les préoccupations de la population, après l'introduction de vaccins, concernant les changements observés dans l'âge de survenue de l'infection, le remplacement du type et la diminution de l'efficacité.

\section{Projets du RCRI concernant la sécurité des vaccins}

Le Réseau de réalisation d'essais cliniques du RCRI, descendant direct du réseau de réalisation d'essais cliniques rapides du PCIRN, possède désormais la formation et l'infrastructure nécessaires pour répondre aux problèmes de santé publique concernant les maladies évitables par la vaccination en établissant des protocoles de recherche de haute qualité pour évaluer les vaccins, les administrer dans un bref délai conformément aux normes réglementaires et internationales et fournir rapidement des résultats sur la sécurité des vaccins à divers moments pendant et après la conduite de l'essai. Les essais prévus pour le cycle de financement de 2014-2017 du RCRI seront axés sur deux importantes maladies évitables par la vaccination : I'hépatite $B$ et la méningococcie invasive (Neisseria meningitidis). L'un des projets vise à déterminer si les jeunes ayant été vaccinés contre l'hépatite B dans leur première enfance présentent toujours des signes sérologiques d'immunité et, dans la négative, si une dose de rappel du vaccin déclencherait la production d'anticorps en raison de l'existence d'une réponse amnestique (mémoire immunitaire). Un deuxième essai clinique à répartition aléatoire, contrôlé et mené à double insu, aura pour but d'évaluer l'innocuité et l'immunogénicité d'un schéma accéléré de vaccination par le vaccin multicomposant contre le méningocoque du sérogroupe $B$. Des critères d'évaluation de la sécurité des vaccins seront incorporés dans les deux études, mesurant notamment les ESSI au point d'injection et systémiques sollicités, les événements indésirables non sollicités et inattendus ainsi que la tolérabilité. Le Réseau de réalisation d'essais cliniques travaillera de concert avec d'autres chercheurs du RCRI pour tester les nouveaux outils de mesure des ESSI, notamment une application pour téléphone intelligent qui facilitera la saisie électronique des données relatives aux ESSI.

La méthode de surveillance utilisée par le Réseau national canadien d'évaluation de la sécurité des vaccins (CANVAS) pour détecter les ESSI survenant après la vaccination contre la grippe convient parfaitement à la surveillance de la sécurité des autres vaccins. Des protocoles visant à adapter le réseau CANVAS pour lui permettre d'assurer la surveillance de la sécurité des nouveaux vaccins, tels que le nouveau vaccin contre le méningocoque de sérogroupe $B$, sont en cours de développement au RCRI.

Le Réseau de cliniques d'immunisation spéciales servira de base à la réalisation d'études cliniques multicentriques concernant la sécurité des vaccins. Au sein du RCRI, le réseau s'intéressera dans un premier temps à la vaccination des patients immunodéprimés. Le premier projet recrutera des enfants atteints de leucémie aiguë lymphoblastique (LAL) ayant accompli une chimiothérapie ou des enfants receveurs d'une greffe de cellules souches. Les objectifs de ce projet sont les suivants : a) décrire les pratiques d'immunisation institutionnelles des centres d'oncologie pédiatrique et de greffe de cellules souches au moyen d'un sondage; $b$ ) cerner les facteurs de risque de faibles titres vaccinaux chez les enfants sous traitement pour une leucémie aiguë lymphoblastique; c) cerner les facteurs cliniques et immunologiques influant sur la réponse vaccinale chez les enfants atteints de LAL et receveurs d'une greffe de cellules souches; et d) déterminer la fréquence des ESSI dans ces deux groupes. L'étude sera menée dans les centres du Réseau de cliniques d'immunisation spéciales selon des méthodes d'évaluation et de suivi des patients semblables à celles mises au point par le PCIRN. Les résultats aideront à appuyer l'établissement de lignes directrices sur l'immunisation pour les enfants atteints de LAL et à harmoniser les pratiques d'immunisation pour les enfants receveurs de greffes de cellules souches. Si la méthode s'avère efficace, son utilisation sera élargie à d'autres populations de patients immunodéprimés. Le 
réseau continuera de consolider sa capacité d'évaluation des signes de problèmes de sécurité pour les nouveaux vaccins et les vaccins émergents, contribuant ainsi à la préparation du pays en cas de pandémie. De futures études pourraient inclure certains ESSI nécessitant un examen en temps réel, l'étude des mécanismes biologiques impliqués dans les ESSI ou l'étude de la base génétique de certains événements particuliers en collaboration avec d'autres réseaux semblables.

L'une des premières études du Réseau collaboratif provincial examinera des problèmes de sécurité pertinents au Canada. Dans le cadre du projet visant à évaluer la sécurité de la vaccination contre le rotavirus au Canada vis-àvis du risque d'invagination à partir de bases de données administratives, les chercheurs prévoient d'adopter une approche pancanadienne fondée sur l'utilisation de données administratives sur la santé afin de déterminer le taux de référence des cas d'invagination chez les nourrissons canadiens et d'établir si l'incidence de cette affection a changé depuis l'introduction des programmes de vaccination contre le rotavirus financés par l'État. Cette étude démontrera la capacité du réseau à mener des études d'innocuité post-commercialisation pertinentes et à fournir des données de référence indispensables au Canada. Elle permettra également d'éclairer la prise de décisions des territoires et provinces qui n'ont pas encore instauré de programmes de vaccination et de fournir des renseignements sur la communication des risques aux parents.

Le Réseau de surveillance des cas graves (SOS) offre une infrastructure importante pour une surveillance ciblée des ESSI, l'évaluation des signes de problèmes d'innocuité et la vérification des hypothèses. Fort d'observateurs formés à la surveillance présents dans tous les hôpitaux participants et ayant déjà des protocoles et des accords de surveillance en place, le réseau est paré pour répondre rapidement aux signes de problèmes d'innocuité émergents en modifiant les protocoles actuels. Tous les observateurs chargés de la surveillance sont parfaitement formés à l'utilisation de la base de données DACIMA actuelle du réseau SOS et le remaniement des éléments de données collectés aux fins de la surveillance des ESSI pourrait être mise en œuvre rapidement afin de permettre à l'ASPC et aux provinces et territoires d'accéder en temps réel aux ESSI observés.

\section{Conclusion}

Le RCRI, comme le PCIRN auparavant, offre une plateforme de recherche nationale multidisciplinaire, collaborative et intégrée pour mener en continu des études de recherche évaluatives financées par l'État et les provinces destinées à éclairer la prise de décisions en matière de santé publique et à fournir l'infrastructure et les capacités nécessaires pour apporter une réponse à l'échelle nationale aux infections nouvelles et émergentes, notamment, entre autres, aux pandémies. Le RCRI jouera également un rôle pivot dans le mentorat des chercheurs en début de carrière, offrant des possibilités aux stagiaires et assurant l'efficacité de la participation des intervenants à tous les stades de la recherche.

\section{Remerciements}

Les auteurs tiennent à remercier tous les investigateurs et le personnel du PCIRN et du RCRI pour leur engagement envers la recherche sur les vaccins.

\section{Conflit d'intérêts}

Scott A. Halperin déclare avoir reçu des subventions des Instituts de recherche en santé du Canada (IRSC) et de l'Agence de la santé publique du Canada (ASPC) durant la conduite de l'étude ainsi que des subventions de plusieurs fabricants de vaccins sans rapport avec les travaux soumis. II participe à des réunions consultatives spéciales de plusieurs fabricants de vaccins. Gaston De Serres déclare avoir reçu des subventions de GlaxoSmithKline sans rapport avec les travaux soumis. Joanne M. Langley déclare avoir reçu des subventions de GlaxoSmithKline durant la conduite de l'étude, des subventions de Sanofi Pasteur sans rapport avec les travaux soumis et avoir participé en tant que bénévole à des comités consultatifs sur les maladies infectieuses et l'immunisation pour le compte du gouvernement de Nouvelle-Écosse et de l'Agence de la santé publique du Canada. Shelly McNeil déclare avoir reçu des subventions de Pfizer, GlaxoSmithKline et Sanofi Pasteur. David Scheifele déclare avoir reçu des subventions de Pfizer, Novartis, GlaxoSmithKline et de Sanofi Pasteur sans rapport avec les travaux soumis. Les autres auteurs n'ont rien a déclarer. 


\section{Financement}

Le PCIRN et le RCRI sont financés par l'Agence de la santé publique du Canada et les Instituts de recherche en santé du Canada.

\section{Références}

(1) Scheifele DW, Ward BJ, Dionne M, Vanderkooi OG, Loeb M, Coleman BL, Li Y, PHAC/CIHR Influenza Research Network (PCIRN). Compatibility of AS03-adjuvanted H1N1pdm09 and seasonal trivalent inactivated influenza vaccines in adults: Results of a randomized, controlled trial. Vaccine. 2012;30(32):4728-32.

(2) Rubinstein E, Predy G, Sauvé L, Hammond GW, Aoki F, Sikora C, et al. The responses of Aboriginal Canadians to adjuvanted pandemic (H1N1) 2009 influenza vaccine. CMAJ. 2011;183(13):e1033-7.

(3) Cooper C, Klein M, Walmsley S, Haase D, MacKinnon-Cameron D, Marty K, et al. on behalf of the PHAC CIHR Influenza Research Network. High-level immunogenicity is achieved vaccine with adjuvanted pandemic $\mathrm{H} 1 \mathrm{~N}_{2}{ }_{2009}$ vaccine and improved with booster dosing in a randomized trial of HIV-infected adults. HIV Clin Trials. 2012;13(1):23-32.

(4) Scheifele DW, Dionne M, Ward BJ, Cooper C, Vanderkooi OG, Li Y, Halperin SA, PHAC/CIHR Influenza Research Network. Safety and immunogenicity of 2010-2011 A/H1N1pdm09-containing trivalent inactivated influenza vaccine in adults previously given AS03-adjuvanted H1N1 2009 pandemic vaccine: Results of a randomized trial. Hum Vaccin Immunother. 2013;9(1):136-43.

(5) Langley JM, Scheifele DW, Quach C, Vanderkooi O, Ward B, McNeil S, et al. Safety and Immunogenicity of 2010-2011 H1N12009-containing trivalent inactivated influenza vaccine in children 12-59 months of age previously given AS03-adjuvanted H1N12009 pandemic vaccine: A PHAC/CIHR Influenza Research Network (PCIRN) study. Vaccine. 2012;30(23):3389-94.

(6) Gagnon R, Primeau MN, Des Roches A, Lemire C, Kagan R, Carr S, et al. PHAC-CIHR Influenza Research Network. Safe vaccination of patients with egg allergy with an adjuvanted pandemic H1N1 vaccine. J Allergy Clin Immunol. 2010;126(2):317-23.

(7) Des Roches A, Paradis L, Gagnon R, Lemire C, Bégin P, Carr S, et al. PCIRN (Public Health Agency of Canada/Canadian Institutes of Health Research Influenza Research Network). Egg-allergic patients can be safely vaccinated against influenza. J Allergy Clin Immunol. 2012;130(5):1213-16.

(8) De Serres G, Gariépy MC, Coleman B, Rouleau I, McNeil S, Benoît M, et al. PHAC-CIHR Influenza Research Network (PCIRN). Short and long-term safety of the 2009 AS03-adjuvanted pandemic vaccine. PLoS One. 2012;7(7):e38563.

(9) Bettinger JA, Vanderkooi OG, MacDonald J, Kellner JD. Rapid online identification of adverse events after influenza immunization in children by PCIRN's National Ambulatory Network. Pediatr Infect Dis J. 2014 ;33(10):1060-4.

(10) Baggs J, Gee J, Lewis E, Fowler G, Benson P, Lieu T, et al. The Vaccine Safety Datalink: A model for monitoring immunization safety. Pediatrics. 2011;127 Suppl 1:S45-53. 